\title{
Passive Spectrum Use and Upcoming Changes in the Spectrum Environment (invited)
}

\author{
Chris van Diepenbeek ${ }^{1}$ \\ Radiocommunications Agency of the Netherlands \\ P.O. Box 450, NL -9700 AL Groningen \\ chris.vdiepenbeek@at-ez.nl
}

The major regulatory instrument for protecting passive spectrum use is the Table of Allocations, together with its footnotes RR 5.340 and 5.149, given in Article 5 of the ITU Radio Regulations. Both technical developments such as Short Range Radar, Ultra Wide Band systems, Cognitive and Software Defined Radio, and the increased recognition of the economic value of radio spectrum, and a desire for more flexibility in the Radio Regulations lead to pressure on passive spectrum use, which may not be perceived as having such a high value for our society. More regulatory instruments and activity is needed to protect in an optimal way the passive use of spectrum. This presentation discusses what administrations in Europe, and more particularly in the Netherlands have done, and what the radio astronomy fraternity could and should do.

RFI mitigation workshop

Groningen, the Netherlands

March 29-31, 2010

\footnotetext{
${ }^{1}$ Speaker
} 


\section{Introduction}

Passive spectrum users have concerns about the increasing commercial pressure on spectrum allocations and assignments, and therefore on the use of spectrum for radio astronomy. A typical example is the introduction of Short Range Radar, also called automotive radar, in the 24 $\mathrm{GHz}$ band. Ultra Wideband applications also use spectrum, which is allocated to the radio astronomy service (RAS).

Moreover one is curious to know whether new technology, such as Cognitive Radio and/or Software Defined Radio, will protect sufficiently the spectrum reserved for the RAS. Flexibility in the Radio Regulations, which is to be discussed at WRC-12, is another topic that raises uncertainty about the required protection of spectrum used by radio astronomers. Before presenting views on these specific applications, it is worthwhile to have a look at the organisations involved in these activities.

\section{The relevant (inter)national organisations for radio astronomy}

\subsection{The ITU}

The International Radio Regulations contain in Article 5, the table of allocations, two specific provisions that are essential for radio astronomy, in addition to specifying which frequency bands are allocated on a primary or secondary basis to the RAS.

- Footnote RR 5.340 is added to frequency bands in which an exclusive allocation is made for passive radio services. It reads: "All emissions are prohibited in the following bands: [list containing 21 frequency bands, ranging from 1.4 to $252 \mathrm{GHz}]$ ". There are only a few geographically limited exceptions made to this list.

- $\quad$ Footnote RR 5.149 appears much more frequently in Article 5. It appears not only in cases where there is a non-exclusive allocation, either on a primary or on a secondary basis, made for the radio astronomy service, but also sometimes in cases where no allocation for radio astronomy is made. RR 5.149 reads: "In making assignments to stations of other services to which the bands [list of 49 frequency bands, ranging from $13.360 \mathrm{kHz}$ to $275 \mathrm{GHz}$ ] are allocated, Administrations are urged to take all practicable steps to protect the radio astronomy service from harmful interference. Emissions from spaceborne or airborne systems can be particularly serious sources of interference to the radio astronomy service (see No's 4.4, 4.6 and Article 29)".

Article 29 contains provisions of a general nature with the objective of giving protection to the RAS. One finds text there stating that Administrations shall cooperate in protection of the service and take all practicable steps to protect the radio astronomy service against interference, e.g. by carefully selecting the output power of transmitters operating in or adjacent to radio astronomy bands, or by selection of locations for observatories. ITU-R has further published various ITU-R Recommendations, e.g. specifying what pfd levels should not be exceeded in order to protect the RAS.

It should be noted that only RR 5.340 has an obligatory character for the ITU Member States, something that RR 5.149 and the various ITU-R Recommendations do not have. Application of these instruments is left to the administrations. Article 29 is very general and does not contain specific protection measures. 
This workshop is about RFI mitigation. I have to note that the attendance of radio astronomers at this workshop is far larger than their attendance at ITU-R meetings, such as WP 7D on Radio Astronomy, TG 1/8 on Short Range Radar and Ultra Wide Band applications, WP 1A on Spectrum above $275 \mathrm{GHz}$, and/or WP 1B on Cognitive Radio, Software Defined Radio and Flexibility. However, a key rule is that prevention of interference is better than mitigation.

ITU texts more or less disregard the fact that a lot of observations, as a result of the Doppler effect, are performed in frequency bands not mentioned as radio astronomy bands in the Radio Regulations. Mitigation techniques discussed in this workshop are of course very relevant to this objective. It does not, however, diminish the need for astronomers to participate in ITU-R meetings with the aim of preventing interference.

\subsection{The CEPT}

CEPT, the European Conference of Postal and Telecommunication Administrations, has 48 members. The European Commission and the European Free Trade Association are Counsellors to CEPT. International Governmental Organizations of States are observers in their own right, and European Telecom, Industry, and Science Organizations are observers based on reciprocal Letters of Understanding (LoU). The major committee within CEPT, relevant to the RAS is the Electronic Communications Committee ${ }^{1}$ (ECC).

The ECC develops common policies and regulations for electronic communications and related applications for Europe, and provides the focal point for information on spectrum use. Its primary objective is to harmonize the efficient use of the radio spectrum, satellite orbits and numbering resources across Europe. The ECC's approach is strategic, open, forward-looking, and based on consensus between member administrations. It applies its expertise in partnership with all stakeholders, the European Commission and ETSI to facilitate the delivery of technologies and services for the benefit of society.

The ECC has four types of deliverables: Decisions, Recommendations, ECC Reports and CEPT Reports. ECC Decisions are regulatory texts providing measures on significant harmonization matters, which CEPT member administrations are strongly urged to follow. ECC Decisions are not obligatory legislative documents; however, they are normally implemented by many CEPT administrations. ECC Recommendations are measures which administrations are encouraged to apply. They are principally intended as harmonization measures for those matters where ECC Decisions are not yet relevant, or as guidance to national administrations. ECC Reports are the result of studies by the ECC, normally developed in support of other, higher level deliverables, i.e. as part of preparation of an ECC Decision or Recommendation. CEPT Reports are responses to mandates from the European Commission.

The European Commission uses the information given in the CEPT Reports and the ECC Decisions as the basis for EC Decisions, for which implementation is obligatory. It is important to mention that all ECC deliverables are subject to a public consultation procedure.

A major subgroup of the ECC is the Conference Preparatory Group (CPG), which develops and agrees the European Common Proposals (ECPs) for the World Radiocommunication Conferences of the ITU. Additionally, it agrees on the Briefs, which contain the reasons and background for the ECPs, as well as views on other proposals.

\footnotetext{
${ }^{1}$ CEPT works with the following definition for Electronic Communications: "transmission, and, where applicable, switching or routing, which permits the conveyance of signals by wire, radio, optical or other electromagnetic means, irrespective of the type of information conveyed".
} 
CEPT is an open and transparent organization. Nearly all meeting documentation is freely accessible. ECC has an LoU with the European Science Foundation (CRAF); it allows CRAF member participation at PT level and organizational representation at WG and ECC Plenary meetings. This means there are four possibilities for CRAF members to influence the ECC: participation as an observer, participation in the national delegation, commenting during consultations ${ }^{2}$, or doing all three !

\subsection{The European Commission}

\subsubsection{The Radio Spectrum Policy Group (RSPG)}

The RSPG is an advisory body for the European Commission. It is composed of high level representatives of the 27 EU Member States, with CEPT/ ECC, and ETSI as observers. The Group assists and advises the Commission on radio spectrum policy issues, on coordination of policy approaches, and, where appropriate, on harmonized conditions with regard to the availability and efficient use of radio spectrum necessary for the establishment and functioning of the internal market.

The RSPG has advised the Commission via so-called Opinions on issues such as Digital Dividend, Streamlining Regulations, Collective Use of Spectrum, Secondary Trading, etc ${ }^{3}$. Two Opinions of the RSPG are of direct relevance for radio astronomers:

(1) RSPG Opinion 6: "A coordinated EU Spectrum approach for Scientific Use of Radio Spectrum", agreed 25-10-'06, has the objective of improving the recognition of scientific use of spectrum. The conclusions of this Opinion are that these services have a considerable societal value, and are incommensurable in financial terms. Intergovernmental commitments have to be a European priority. Exclusive allocations are needed only for a limited portion of the spectrum. For non RR No 5.340 bands, in case a sharing scenario has to be decided upon, the impact on both services shall be assessed.

There is a relation between this Opinion 6 and the WRC-12 agenda 8.1.1 item C. This agenda item requires the Director of the Bureau Radiocommunications to report on sector activities related to Resolution 673 (WRC-07): "Radiocommunications use for Earth observation applications". The objective of this Resolution is to improve the recognition of this type of spectrum use. It is interesting to observe that Resolution 673 does not contain a word about the RAS, and the question arises as to why the RAS, facing a comparable problem, missed the opportunity of being mentioned there.

In response to Resolution 673, the Radiocommunications Agency of the Netherlands initiated the development of the ITU-R Report; "The essential role and global importance of radio spectrum use for Earth observations and for related applications". The related applications here are the radio astronomy and the space research services. Again, I have to make the comment that initially radio astronomers paid less attention to the development of text for the draft Report than their colleagues in Earth observation organizations. This leads me to the suggestion that there should be much closer collaboration between these two science communities: instead of using spectrum for two or three separate 'Science Services', but for a more familial "Observation Services".

\footnotetext{
${ }^{2}$ see: www.ero.dk

${ }^{3}$ see: http://rspg.groups.eu.int
} 
(2) RSPG Opinion 12: "Best Practices Regarding the Use of Spectrum by Some Public Sectors" was agreed 11-2-'09. Its objective is to identify best practices for a more efficient use of spectrum by public sector bodies in the areas of defense, emergency \& public safety, and public transport, with a view to assisting spectrum management authorities in EU Member States to ensure that these public sector bodies perform their task effectively, and that scarce radio frequencies are not under-utilized. Spectrum use by radio astronomy is included in use by the public sector. The opinion describes multiple approaches:

- Document the rights of use by public sector bodies;

- $\quad$ Determine needs via ex-ante detailed audit;

- $\quad$ Acquire service from the market, introduce spectrum pricing etc.;

- Apply a "justification procedure"; public sector bodies have to submit a detailed explanation of their spectrum requirements.

The spectrum management authorities in the Netherlands will apply the method presented under bullet 4 (see section 2.4). I consider this approach to be a very interesting one for the RAS.

Draft Opinions of the Radio spectrum policy Group are subject to a Public Consultation and are published on < http://rspg.groups.eu.int $>$. This creates another opportunity to react and express your views.

\subsubsection{Radio Spectrum Committee (RSC)}

The aim of the RSC is to establish a policy and legal framework in the Community in order to ensure the coordination of policy approaches and, where appropriate, harmonized conditions with regard to the availability and efficient use of the radio spectrum necessary for the establishment and functioning of the internal market in Community policy areas such as electronic communications, transport, and research \& development ${ }^{4}$.

For the development of technical implementing measures which fall within the remit of the CEPT, such as the harmonization of radio frequency allocations and of information availability, the Commission shall issue mandates to the CEPT, setting out the tasks to be performed and their timetable.

Within the ECC, consensus is the preferred basis for EC decisions, though weighted voting is possible. However, the implementation of EC Decisions is obligatory for the Member States. The Commission uses the CEPT Report (and ECC Decisions) as the technical basis for its Decisions. There is no public consultation for EC Decisions, but we should note that the CEPT Report has undergone a Public Consultation. It is therefore important for radio astronomers, when they have a concern about a certain issue, to influence the CEPT Report by participating in the ECC work, or by directly lobbying the participants to the ECC meeting or the Commission.

\subsection{The Netherlands}

The Dutch spectrum management authorities intend to apply in the near future the principle, mentioned above in section 2.3.2, that public spectrum users have to justify their needs on a regular basis. Once the envisaged change in the telecommunication law has been approved by parliament and enters into force, the Science Services will also have to submit every 3 years a

\footnotetext{
${ }^{4}$ ECC and EC Decisions related to the internal market electronic communications, transport, and R \&D can be found at: www.ero.dk and http://ec.europa.eu/information_society/policy/ecomm/radio_spectrum
} 
plan containing their justified needs. Under that regime the minister of Education and Science will specify for radio astronomy matters such as:

- The purpose and legal basis for the public use,

- The organization to use the spectrum,

- An inventory of required spectrum,

- The time, location, and conditions for its use,

Of course, the minister responsible for telecommunication matters has the right to request further information.

This approach is considered to remove and relieve commercial pressure on the use of spectrum by radio astronomy. You are invited to evaluate this procedure. If you wish, you could develop an ITU-R Report on it in order to aid in the proliferation of this approach, and to inform other concerned parties.

\section{Electronic Communications in ECC and EC}

\subsection{Short Range Radar in 24 GHz Band}

Noting that the USA has adopted a permanent regulation for SRR at $24 \mathrm{GHz}$, and taking into account developments in the car industry, both the ECC and the EC have adopted an interim regulation on $24 \mathrm{GHz}$ in Europe (until 1 July 2013$)^{5}$, while being aware that it is very difficult to enforce this regulation to protect radio astronomy. However, as there is no take-off at the moment, the car industry has requested an extension of the interim regulations.

There is still considerable disagreement in CEPT concerning the regulations for the near future: either to prolong for a limited period the use at $24 \mathrm{GHz}$, or, as an alternative, at $26-29 \mathrm{GHz}$ while allowing a maximum density of 7 or $10 \%$ respectively before a move to $77-81 \mathrm{GHz}$, or alternately to move directly to $77-81 \mathrm{GHz}$ once the agreed interim solution has expired. It should be noted that there are no interference reports at $24 \mathrm{GHz}$.

ECC has, in response to EC Mandates, developed CEPT Report 36 on $24 \mathrm{GHz}$, but its approval is on hold until a final ECC decision on the use of the $26-29 \mathrm{GHz}$ band. CEPT report 37 on the use of the $26-29 \mathrm{GHz}$ band is in public consultation at the time of this writing. So you still have an opportunity to express your views.

At this time the provisional conclusions within the ECC are that the existing regulation for SRR $24 \mathrm{GHz}$ systems should in principle be kept without substantial changes, while taking into account the resulting constraints for coexistence between SRR $24 \mathrm{GHz}$ on the one hand, and EESS, FS \& Radio Astronomy on the other. There is a consensus that future SRR applications should operate at $77-81 \mathrm{GHz}$.

\subsection{Cognitive Radio (CR) Software Defined Radio (SDR)}

ECC CPG takes the view that there shall be no definitions for CR or SDR in the Radio Regulations. This is based on the general principle that any radio system may implement

\footnotetext{
${ }^{5}$ Decisions on SRR: ECC/DEC(04)03 (77-81 GHz), ECC/Dec(04)10 amended (24 GHz temporarily), 2004/545/EC (77-81 GHz), and 2005/50/EC (24 GHz temporarily).
} 
SDR/CR techniques within any radiocommunication service as long as it operates in accordance with the provisions of the Radio Regulations applicable for that specific service in the related frequency band. Frequencies or frequency bands (tuning range) for specific applications could be harmonized, as necessary, on a world wide basis in ITU-R Recommendations or regionally.

No regulatory actions would be required for SDR .

CPG foresees the following deployment scenarios:

- If all systems in a certain band have CR capabilities, then CR can share on a primary basis.

- In case of sharing in a band with systems not having CR capability: CR use is secondary to the use of the service having the primary allocation.

This leads to the conclusion that there is no need to exclude radio astronomy bands.

\subsection{Flexibility (Resolution 951, WRC-07)}

The ECC has adopted a policy to increase flexibility in the Radio Regulations while ensuring compatibility between services: allocations should be done in the broadest possible way under provisions to ensure technological neutrality where possible. Therefore, one should include in the Radio Regulations only issues which have an international implication: the use of a frequency band can be decided at national or regional level, in accordance with the Radio Regulations, noting that some use is international, or has international implications.

ECC CPG is developing a WRC Resolution on principles for enhancing the international spectrum regulatory framework, taking into account:

- $\quad$ the current trend towards convergence of applications within different services;

- $\quad$ the need for Regulations to keep pace with developments, while ensuring effective and efficient use of spectrum allowing operations to be free of harmful interference;

- that modifications to the Radio Regulations may take 4-8 years, and sometimes longer.

ECC considers that national licensing is the appropriate and best tool to protect the RAS. Within ITU-R some progress has been made with regard to the convergence of fixed and mobile services by reviewing the definitions for these services. The debate about flexibility raises the question whether one should also consider it applicable to the RAS. In Article 5 of the Radio regulations there are 20 frequency bands allocated to both EESS (passive), Radio Astronomy and SRS (passive). Would there be an advantage in having an allocation common to these services defined as "Observation Service (passive)"?

\section{Conclusion}

To create optimal spectrum usage conditions for the RAS it is very important that the collaboration between spectrum management and radio astronomy organizations is optimal. In my presentation I have encouraged you to participate more actively in developments related to spectrum management. I invite you, therefore, to look a little bit less at the Universe, and look a little bit more at the spectrum! 